\title{
Musculoskeletal imaging of a painful knee: Diagnosis
}

\section{S K Misser, MB ChB, FCRad (D)}

Lake Smit and Partners, Durban

Corresponding author: S Misser (shalendramisser@hotmail.com)
We congratulate Dr Dirk van der Merwe of Drs Schnetler, Corbett and Partners, Cape Town, for a detailed diagnosis for which he receives an award of R1 000 from the RSSA. Dr Misser elaborates below on the condition and its imaging. Please refer to page 39 of the February 2012 issue of the SAJR (http://www.sajr.org.za/index.php/sajr/article/ view/691/549) for the presentation details.

In addition, delayed congratulations to Dr Rory Tayler, who is a consultant in the Department of Radiology at $\mathrm{CH}$ Baragwanath Hospital, for submitting a precise diagnosis of the December 2011 quiz case, for which he also receives a R1000 award from the RSSA. His submission fell within the stipulated cut-off date but missed publication because of the earlier-than-usual printing of the SAJR. Dr Misser elaborated on this case in the February 2012 issue of the journal.

\section{Presentation and diagnosis}

(The images referred to below are not reproduced here but are in the online February 2012 SAJR at the above URL.)

The plain radiographs (Figs 1 and 2) demonstrate moderate background osteopaenia and osteoarthritic change involving both compartments of the femoro-tibial joint. Fig. 2 (performed at time of admission) reveals microfracture of the medial tibial plateaux with collapsed subchondral bony plate. MR images (Figs 3 - 6) show a degenerative medial meniscus tear with joint fluid and established osteoarthritic change. Articular surface hyperintensity of both femoral condyles and the medial tibial condyle with subchondral fracture at the latter is noted. The combination of features is in keeping with subchondral insufficiency microfracture or previously described spontaneous osteonecrosis of the knee (SONK).

First described in 1968 by Ahlbach et al., ${ }^{1}$ this entity has become a distinct form of osteonecrosis with typical clinical and radiological patterns. Generally, elderly female patients, with no preceding steroid or ethanol use, present with acute knee pain along the joint line. The differentiation from secondary osteonecrosis may be difficult on imaging alone and predisposing factors, patient profile and areas of joints involved are useful distinguishing features (see box below). ${ }^{2}$
SONK has been categorised into 5 radiographic stages. ${ }^{3}$ In the immediate phase, plain radiographs are usually normal (stage 1 ). In stage 2, flattening of the weight-bearing surface of the femoral condyle is noted. Follow-up radiographs may demonstrate some progressive articular surface change with lucent subchondral focus (stage 3 ). The focus of osteonecrosis enlarges and becomes surrounded by a sclerotic halo in stage 4. Development of secondary osteoarthritic changes including osteophytes, erosions, sclerosis and joint space narrowing, as well as later tibial articular surface involvement, marks stage 5 disease. ${ }^{3}$

Fat-suppressed MRI studies, with long TR sequences (T2- and PD-weighted) characteristically demonstrate high signal intensity in the condyles with a central subchondral low signal area. ${ }^{2}$ This is classically described as the bone marrow oedema pattern with central focal lowintensity necrotic zone.

Technetium-labelled isotope bone scan demonstrates focal increased tracer uptake at the affected condyle. Scintigraphy was used by Ahlbäck ${ }^{1}$ in the landmark description of SONK by showing increased tracer uptake localised to one side of the joint after excluding other predisposing factors.

More recently, with histopathological correlation, the primary event leading to SONK was found to be a subchondral insufficiency fracture, and the resultant osteonecrosis was due to the microfracture. ${ }^{2}$ This has led to the new descriptive terminology for this entity with improved reference to both the aetiology and variability of the outcome in patients with SONK. The importance of detection of this process on imaging studies lies in the fact that, owing to its subchondral location, the lesion may not be detectable at arthroscopy. The radiologist is therefore instrumental in making this diagnosis, which may be occult to the orthopaedic surgeon's arthroscope.

1. Ahlbäck S, Bauer GC, Bohne WH. Spontaneous osteonecrosis of the knee. Arthrit Rheumat 1968;11:705733.

2. Yamamoto T, Bullough PG. Spontaneous osteonecrosis of the knee: The result of subchondral insufficiency fracture. J Bone Joint Surg 2000;82:858-866.

3. Stoller DW. Magnetic Resonance Imaging in Orthopaedics and Sports Medicine. 3rd ed. Vol 1. Philadelphia: Lippincott Williams and Wilkins, 2007:685-692.

\begin{tabular}{lll} 
& SONK & Atraumatic osteonecrosis \\
\hline Synonyms & Primary necrosis, osteonecrosis & Secondary, idiopathic osteonecrosis \\
Age group & Older patients, usually $>55$ years & Younger patients, usually $<55$ years \\
Patient profile & Elderly female, with moderate OA & Young active \\
Presentation & Acute pain/acute-on-chronic pain & Insidious onset and slow progression \\
Risk factors & None & Steroid/ethanol use, renal disease \\
Joints involved & Weight-bearing, usually unilateral & Non-weight-bearing, commonly bilateral \\
Location of bone involvement & Epiphyseal to subchondral surface & Diaphyseal, metaphyseal, epiphyseal \\
& Increased signal at margin, decreased signal at & Decreased signal at margin with increased signal \\
T2-weighted MRI & centre of lesion &
\end{tabular}

